\title{
A numerically scalable domain decomposition method for the solution of frictionless contact problems
}

\author{
D. Dureisseix, C. Farhat ${ }^{\dagger}$
}

\begin{abstract}
We present a domain decomposition method with Lagrange multipliers for solving iteratively frictionless contact problems. This method, which is based on the FETI method and therefore is named here the FETI$\mathrm{C}$ method, incorporates a coarse contact system that guides the iterative prediction of the active zone of contact. We demonstrate numerically that this method is numerically scalable with respect to both the problem size and the number of subdomains.

This article in its final form was published in International Journal for Numerical Methods in Engineering, Volume 50, Issue 12, 2001, Pages 2643-2666. Wiley

doi:10.1002/nme.140
\end{abstract}

keyword contact; domain decomposition; FETI; scalability

\section{INTRODUCTION}

Contact problems are frequent in structural analysis, particularly when investigating the assembly of substructures. They are characterized by constraints such as non-penetration conditions, and an active area of contact - that is, an area where contact effectively occurs - that is unknown a priori. For these reasons, these problems lead to stiff non-linear systems of equations. Even in the absence of friction, several approaches exist for solving static contact problems $[20,34,32]$. In most of them, the numerical methods that are employed for enforcing the contact constraints can be grouped into Lagrange multiplier and penalty methods [33].

The penalty methods $[19,2]$ are closely related to the regularization of the contact constraints. They are usually formulated in terms of the displacement

*LMT-Cachan (École Normale Supérieure de Cachan/CNRS/Université Paris 6), 61 Avenue du Président Wilson, F-94235 Cachan CEDEX, FRANCE

${ }^{\dagger}$ Corresponding author, Department of Aerospace Engineering Sciences and Center for Aerospace Structures, University of Colorado, Campus Box 429, Boulder, CO 80309-0429, USA 
variables, and therefore are primal methods. They allow treating contact as a material behavior, as examplified by the method of joint finite elements [1]. Penalty methods can experience various numerical difficulties, especially illconditioning, when a too large or too small penalty parameter is introduced.

Lagrange multiplier methods are dual methods where the multipliers, which represent the contact reaction forces, are introduced in order to enforce exactly the non-penetration conditions. These methods can be further classified into (a) trial and error approaches for determining the active zone of contact, (b) mathematical programming techniques [21, 18], (c) search gradient methods $[25,10,27,28]$, and (d) a combination of all of these approaches. Augmented Lagrange multiplier methods $[3,22,26,6]$ result in mixed formulations involving both displacement and force unknowns. The numerical solution schemes underlying both the Lagrange multiplier and augmented Lagrange multiplier methods are often related to the Uzawa algorithm [4, 31, 8].

Recently, various forms of domain decomposition have been introduced in the formulation of contact problems in order to speedup the solution of these problems, particularly when they are large-scale, on both serial and parallel computing platforms $[7,11,5]$. Of particular interest is the approach proposed in [11], not only because of its numerous innovative concepts, but also because it is based on the FETI domain decomposition method [12, 16, 17], which has well established numerical scalability properties for both second-order elasticity $[15,23]$ and fourth-order plate and shell problems [14, 13, 24]. We remind the reader that an iterative method is said to be numerically scalable if its convergence properties - for example, the number of iterations for convergence - are asymptotically independent of the size of the problem to be solved.

The objective of this paper is to present an alternative domain decomposition method for the solution of frictionless contact problems that is also based on the FETI method, and which for this reason is named the FETI-C method (as in FETI-Contact).

FETI-C is in many aspects different from the domain decomposition method recently proposed in [11] for solving frictionless contact problems. Indeed, the solution strategy described in [11] is organized around two levels of iterations, the first one aimed at satisfying the contact conditions, and the second one at satisfying, among other things, the equilibrium conditions. In the outeriterations, an active zone of contact is updated by a mathematical programming technique. In the inner-iterations, a minimal subregion of the previously predicted area of contact is frozen and a state of equilibrium is sought after. In the FETI-C method described in this paper, both contact and equilibrium conditions are updated by a single iterative procedure. Most importantly, the FETI-C method incorporates an auxiliary "coarse contact problem" which not only guides the prediction of the active zone of contact, but also appears to ensure the numerical scalability of the proposed solution method with respect to both the number of subdomains, and the size of the problem. These numerical scalability properties are demonstrated numerically in this paper.

For simplicity, and without any loss of generality, we consider here only the case where the subdomains in contact have matching discrete interfaces. 
We also assume that all structural components undergo small deformations as well as small displacements and rotations. In Section 2, we review the FETI method in order to keep this paper as self-contained as possible. In Section 3, we overview the formulation of the frictionless contact problem. In Section 4, we highlight the suitability of the FETI framework to the solution of contact problems, and describe the FETI-C non-linear solver. In Section 5, we validate this solver using sample two- and three-dimensional frictionless contact problems and demonstrate numerically its numerical scalability with respect to both the problem size and the number of subdomains. Finally, we conclude this paper in Section 6 .

\section{AN OVERVIEW OF THE FETI METHOD}

\subsection{Domain decomposition with Lagrange multipliers}

The FETI method $[12,16,17,14,13]$ is a non-overlapping domain decomposition or substructuring method with Lagrange multipliers. It was originally developed for solving efficiently on parallel processors large-scale linear or linearized systems of equations arising from the discretization of structural mechanics problems defined on a domain $\Omega$. Such a system of equations can be written as

$$
\boldsymbol{K}_{g} \boldsymbol{u}_{g}=\boldsymbol{f}_{g}
$$

where for a large class of applications, $\boldsymbol{K}_{g}$ is a symmetric positive definite or semi-definite matrix constructed from the assembly of element level stiffness matrices by introducing a global numbering of the degrees of freedom (d.o.f.) indicated here by the subscript $g, \boldsymbol{u}_{g}$ is the vector of generalized displacements, and $\boldsymbol{f}_{g}$ the vector of prescribed forces. In the FETI method, the given domain $\Omega$ is partitioned into $N_{s}$ non-overlapping subdomains $\Omega^{(s)}$, the equilibrium equations are written at the subdomain level, and Lagrange multipliers are introduced at the subdomain interfaces to enforce the compatibility of the displacement field. In such an approach, the initial mechanical problem is essentially reformulated as a saddle point problem whose Euler equations are given by [12]

$$
\delta_{\boldsymbol{v}^{(s)}, \boldsymbol{\mu}} \mathcal{L}\left(\boldsymbol{v}^{(s)}, \boldsymbol{\mu}\right)=0
$$

where $\delta_{\boldsymbol{v}^{(s)}, \boldsymbol{\mu}}$ designates the variation with respect to the arguments $\boldsymbol{v}^{(s)}$ and $\boldsymbol{\mu}$, and $\mathcal{L}$ is the Lagrangian of the problem and can be written as

$$
\mathcal{L}\left(\boldsymbol{v}^{(s)}, \boldsymbol{\mu}\right)=\sum_{s=1}^{N_{s}}\left(\frac{1}{2} \boldsymbol{v}^{(s)^{T}} \boldsymbol{K}^{(s)} \boldsymbol{v}^{(s)}-\boldsymbol{v}^{(s)^{T}} \boldsymbol{f}^{(s)}\right)+\boldsymbol{\mu}^{T} \sum_{s=1}^{N_{s}} \boldsymbol{B}^{(s)} \boldsymbol{v}^{(s)}
$$

Here, the superscript $s$ designates a subdomain quantity, the superscript $T$ denotes the transpose of a quantity, $\boldsymbol{v}^{(s)}$ and $\boldsymbol{\mu}$ are some admissible subdomain displacement and Lagrange multiplier fields, respectively, $\boldsymbol{K}^{(s)}$ is the subdomain 


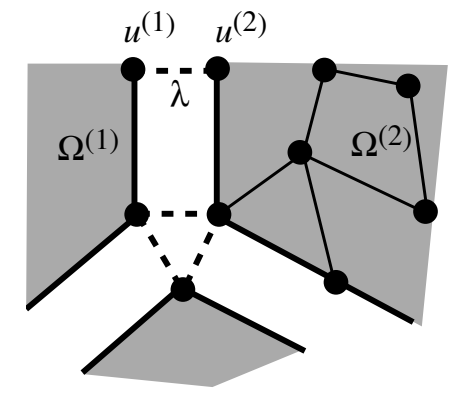

Figure 1: Interface assembly in the FETI formulation

stiffness matrix, $\boldsymbol{f}^{(s)}$ the subdomain vector of prescribed forces, and $\boldsymbol{B}^{(s)}$ the signed Boolean matrix that extracts from a subdomain vector $\boldsymbol{v}^{(s)}$ its signed $( \pm)$ restriction to the subdomain interface boundary. The Euler equations (2) lead to the following constrained problem which is equivalent to problem (1)

$$
\begin{aligned}
\boldsymbol{K}^{(s)} \boldsymbol{u}^{(s)} & =\boldsymbol{f}^{(s)}-\boldsymbol{B}^{(s)^{T}} \boldsymbol{\lambda} \quad s=1, \cdots, N_{s} \\
\sum_{s=1}^{N_{s}} \boldsymbol{B}^{(s)} \boldsymbol{u}^{(s)} & =\mathbf{0}
\end{aligned}
$$

where $\boldsymbol{u}^{(s)}$ and $\boldsymbol{\lambda}$ are respectively the subdomain displacements and Lagrange multipliers that are the stationary points of $\mathcal{L}$. The mechanical interpretation of equations (4) and (5) is graphically depicted in Figure 1: each of equations (4) expresses the subdomain equilibrium under the action of the prescribed forces $\boldsymbol{f}^{(s)}$ and the subdomain interface forces $\boldsymbol{B}^{(s)^{T}} \boldsymbol{\lambda}$, and equation (5) expresses the compatibility of the subdomain displacement fields at the subdomain interfaces. There is one compatibility equation for each pair of d.o.f. that connect at a subdomain interface. Once $\boldsymbol{\lambda}$ is determined, the subdomain displacement fields can be recovered by solving concurrently the equilibrium equations to obtain

$$
\boldsymbol{u}^{(s)}=\boldsymbol{K}^{(s)^{+}}\left(\boldsymbol{f}^{(s)}-\boldsymbol{B}^{(s)^{T}} \boldsymbol{\lambda}\right)+\boldsymbol{R}^{(s)} \boldsymbol{\alpha}^{(s)}
$$

where $\boldsymbol{K}^{(s)^{+}}$denotes the inverse of $\boldsymbol{K}^{(s)}$ if $\Omega^{(s)}$ has sufficient Dirichlet boundary conditions to prevent $\boldsymbol{K}^{(s)}$ from being singular, or a generalized inverse of $\boldsymbol{K}^{(s)}$ if $\Omega^{(s)}$ is a floating subdomain - that is, a subdomain without sufficient displacement boundary conditions to prevent $\boldsymbol{K}^{(s)}$ from being singular. In the latter case, the columns of $\boldsymbol{R}^{(s)}$ represent the rigid body modes of $\Omega^{(s)}$, i.e. $\boldsymbol{R}^{(s)}=\operatorname{ker} \boldsymbol{K}^{(s)}$, and $\boldsymbol{\alpha}^{(s)}$ is the set of amplitudes that specifies the contribution of the null space $\boldsymbol{R}^{(s)}$ to the solution $\boldsymbol{u}^{(s)}$. These coefficients can be determined by requiring that each subdomain problem be mathematically solvable - that is, each floating subdomain be self-equilibrated — which can be written as

$$
\boldsymbol{R}^{(s)^{T}}\left(\boldsymbol{f}^{(s)}-\boldsymbol{B}^{(s)^{T}} \boldsymbol{\lambda}\right)=\mathbf{0}
$$


Substituting equation (6) into the compatibility equation (5) and exploiting the solvability condition (7) transforms the constrained problem $(4,5)$ into the interface problem

$$
\left[\begin{array}{cc}
\boldsymbol{F}_{I} & -\boldsymbol{G}_{I} \\
-\boldsymbol{G}_{I}^{T} & \mathbf{0}
\end{array}\right]\left[\begin{array}{l}
\boldsymbol{\lambda} \\
\boldsymbol{\alpha}
\end{array}\right]=\left[\begin{array}{c}
\boldsymbol{d} \\
-\boldsymbol{e}
\end{array}\right]
$$

where

$$
\begin{aligned}
\boldsymbol{F}_{I} & =\sum_{s=1}^{N_{s}} \boldsymbol{B}^{(s)} \boldsymbol{K}^{(s)^{+}} \boldsymbol{B}^{(s)^{T}} \\
\boldsymbol{d} & =\sum_{s=1}^{N_{s}} \boldsymbol{B}^{(s)} \boldsymbol{K}^{(s)^{+}} \boldsymbol{f}^{(s)} \\
\boldsymbol{G}_{I} & =\left[\begin{array}{lll}
\boldsymbol{B}^{(1)} \boldsymbol{R}^{(1)} & \cdots & \boldsymbol{B}^{\left(N_{s}\right)} \boldsymbol{R}^{\left(N_{s}\right)}
\end{array}\right] \\
\boldsymbol{\alpha} & =\left[\begin{array}{lll}
\boldsymbol{\alpha}^{(1)^{T}} & \cdots & \boldsymbol{\alpha}^{\left(N_{s}\right)^{T}}
\end{array}\right]^{T} \\
\boldsymbol{e} & =\left[\begin{array}{llll}
\boldsymbol{f}^{(1)^{T}} \boldsymbol{R}^{(1)} & \cdots & \boldsymbol{f}^{\left(N_{s}\right)^{T}} \boldsymbol{R}^{\left(N_{s}\right)}
\end{array}\right]^{T}
\end{aligned}
$$

Because $\boldsymbol{\lambda}$ is a dual variable to the primal variables $\boldsymbol{u}^{(s)}$, the interface problem (8) is called a dual interface problem. This interface problem is best solved by an iterative algorithm, the choice of which completes the description of the original FETI method.

\subsection{Iterative solution of the dual interface problem}

In the FETI method, the interface problem (8) is solved by a preconditioned conjugate projected gradient (PCPG) algorithm. More specifically, the indefinite interface problem (8) is transformed into a semi-definite system of equations by eliminating the self-equilibrium condition $\boldsymbol{G}_{I}^{T} \boldsymbol{\lambda}=\boldsymbol{e}$ using the splitting

$$
\lambda=\lambda^{0}+P(Q) \bar{\lambda}
$$

where $\boldsymbol{\lambda}^{0}$ is a particular solution of $\boldsymbol{G}_{I}^{T} \boldsymbol{\lambda}=\boldsymbol{e}$ of the form

$$
\lambda^{0}=\boldsymbol{Q} \boldsymbol{G}_{I}\left(\boldsymbol{G}_{I}^{T} \boldsymbol{Q} \boldsymbol{G}_{I}\right)^{-1} \boldsymbol{e}
$$

and $\boldsymbol{P}(\boldsymbol{Q})$ is a projection matrix defined for any given matrix $\boldsymbol{Q}$ by

$$
\boldsymbol{P}(\boldsymbol{Q})=\boldsymbol{I}-\boldsymbol{Q} \boldsymbol{G}_{I}\left(\boldsymbol{G}_{I}^{T} \boldsymbol{Q} \boldsymbol{G}_{I}\right)^{-1} \boldsymbol{G}_{I}^{T}
$$

Note that for any matrix $\boldsymbol{Q}, \boldsymbol{G}_{I}^{T} \boldsymbol{P}(\boldsymbol{Q})=\mathbf{0}$.

Applying the splitting (10) to the interface problem (8) leads to the alternative symmetric positive semi-definite interface problem

$$
\boldsymbol{P}(\boldsymbol{Q})^{T} \boldsymbol{F}_{I} \boldsymbol{P}(\boldsymbol{Q}) \overline{\boldsymbol{\lambda}}=\boldsymbol{P}(\boldsymbol{Q})^{T}\left(\boldsymbol{d}-\boldsymbol{F}_{I} \boldsymbol{\lambda}^{0}\right)
$$


which can be solved by a standard PCG algorithm.

Two different preconditioners have been proposed: the mathematically optimal Dirichlet preconditioner $\overline{\boldsymbol{F}}_{I}^{D^{-1}}$ introduced in [15], and the computationally economical lumped preconditioner $\overline{\boldsymbol{F}}_{I}^{L^{-1}}$ proposed in earlier works $[12,16]$. If each subdomain stiffness matrix is partitioned as

$$
\boldsymbol{K}^{(s)}=\left[\begin{array}{cc}
\boldsymbol{K}_{i i}^{(s)} & \boldsymbol{K}_{i b}^{(s)} \\
\boldsymbol{K}_{i b}^{(s)^{T}} & \boldsymbol{K}_{b b}^{(s)}
\end{array}\right]
$$

where the subscripts $i$ and $b$ designate the subdomain interior and interface boundary d.o.f., respectively, then the Dirichlet preconditioner can be written as

$$
\overline{\boldsymbol{F}}_{I}^{D^{-1}}=\boldsymbol{W}\left(\sum_{s=1}^{N_{s}} \boldsymbol{B}^{(s)}\left[\begin{array}{cc}
\mathbf{0} & \mathbf{0} \\
\mathbf{0} & \boldsymbol{S}_{b b}^{(s)}
\end{array}\right] \boldsymbol{B}^{(s)^{T}}\right) \boldsymbol{W}
$$

and the lumped preconditioner is given by

$$
\overline{\boldsymbol{F}}_{I}^{L^{-1}}=\boldsymbol{W}\left(\sum_{s=1}^{N_{s}} \boldsymbol{B}^{(s)}\left[\begin{array}{cc}
\mathbf{0} & \mathbf{0} \\
\mathbf{0} & \boldsymbol{K}_{b b}^{(s)}
\end{array}\right] \boldsymbol{B}^{(s)^{T}}\right) \boldsymbol{W}
$$

In the above expressions of $\overline{\boldsymbol{F}}_{I}^{D^{-1}}$ and $\overline{\boldsymbol{F}}_{I}^{L^{-1}}, \boldsymbol{W}$ is a diagonal matrix storing in each of its entries the inverse of the multiplicity of an interface d.o.f. [17, 14] - that is, the inverse of the number of subdomains to which an interface d.o.f. belongs - and $\boldsymbol{S}_{b b}^{(s)}$ is the following subdomain primal Schur complement

$$
\boldsymbol{S}_{b b}^{(s)}=\boldsymbol{K}_{b b}^{(s)}-\boldsymbol{K}_{i b}^{(s)^{T}} \boldsymbol{K}_{i i}^{(s)^{-1}} \boldsymbol{K}_{i b}^{(s)}
$$

Note that $\boldsymbol{K}_{i i}^{(s)}$ is non-singular since it corresponds to the system matrix with all interface boundaries fixed and thus $\boldsymbol{K}_{i i}^{(s)^{-1}}$ exists. Both Dirichlet and lumped preconditioners have been recently extended in [29] for addressing more efficiently heterogeneous problems. In particular, it was shown in [29] that such extensions can be simply designed by redefining appropriately the scaling matrix $W$.

The iterative FETI solver with $\boldsymbol{Q}=\boldsymbol{I}$ is summarized in Table 1. A more detailed description of this algorithm can be found in [17].

\section{Remarks}

- For most problems, the simplest choice $\boldsymbol{Q}=\boldsymbol{I}$ is computationally the most effective one. Choosing $\boldsymbol{Q}=\boldsymbol{Q}^{D}=\overline{\boldsymbol{F}}_{I}^{D^{-1}}$ or $\boldsymbol{Q}=\boldsymbol{Q}^{L}=\overline{\boldsymbol{F}}_{I}^{L^{-1}}$ is recommended for heterogeneous problems [17]. 
Table 1: The FETI PCPG algorithm

\begin{tabular}{|c|c|c|c|}
\hline Initialize & & & \\
\hline & $\lambda^{0}$ & $=$ & $\boldsymbol{G}_{I}\left(\boldsymbol{G}_{I}^{T} \boldsymbol{G}_{I}\right)^{-1} \boldsymbol{e}$ \\
\hline & & $=$ & $\boldsymbol{d}-\boldsymbol{F}_{I} \boldsymbol{\lambda}^{0}$ \\
\hline Iterate $k=1,2$, & . until & conv & rgence \\
\hline Project & $\boldsymbol{w}^{k-1}$ & $=$ & $\boldsymbol{P}^{T} \boldsymbol{r}^{k-1}$ \\
\hline Precondition & $z^{k-1}$ & $=$ & $\overline{\boldsymbol{F}}_{I}^{-1} \boldsymbol{w}^{k-1}$ \\
\hline Re-project & $\boldsymbol{y}^{k-1}$ & $=$ & $\boldsymbol{P} \boldsymbol{z}^{k-1}$ \\
\hline Conjugate & $\zeta^{k}$ & $=$ & $\frac{\boldsymbol{y}^{k-1^{T}} \boldsymbol{w}^{k-1}}{k^{k} T^{T}-2} \quad\left(\zeta^{1}=0\right)$ \\
\hline & $p^{k}$ & $=$ & $\begin{array}{l}\boldsymbol{y}^{k-2^{T}} \boldsymbol{w}^{k-2} \\
\boldsymbol{y}^{k-1}+\zeta^{k} \boldsymbol{p}^{k-1} \quad\left(\boldsymbol{p}^{1}=\boldsymbol{y}^{0}\right)\end{array}$ \\
\hline Minimize & $\eta^{k}$ & $=$ & $\boldsymbol{p}^{k-1^{T}} \boldsymbol{w}^{k-1}$ \\
\hline & $\pi$ & & $\overline{p_{k-1}^{k^{T}} \boldsymbol{F}_{I} \boldsymbol{p}_{k k_{k}^{k}}}$ \\
\hline $\begin{array}{r}\text { Update } \\
\text { Compute residual }\end{array}$ & $\begin{array}{l}\lambda^{n} \\
r^{k}\end{array}$ & $\begin{array}{l}= \\
=\end{array}$ & $\begin{array}{l}\boldsymbol{\lambda}^{n-1}+\eta^{n} \boldsymbol{p}^{n} \\
\boldsymbol{r}^{k-1}-\eta^{k} \boldsymbol{F}_{I} \boldsymbol{p}^{k}\end{array}$ \\
\hline
\end{tabular}

- From Eqs. $(6,9,12)$ and Table 1, it follows that

$$
\begin{aligned}
\sum_{s=1}^{N_{s}} \boldsymbol{B}^{(s)} \boldsymbol{u}^{(s)} & =\boldsymbol{d}-\boldsymbol{F}_{I} \boldsymbol{\lambda}+\boldsymbol{G}_{I} \boldsymbol{\alpha} \\
& =\boldsymbol{r}+\boldsymbol{G}_{I} \boldsymbol{\alpha} \\
& =\boldsymbol{P}^{T} \boldsymbol{r} \\
& =\boldsymbol{w}
\end{aligned}
$$

which shows that at each iteration $k$, the projected residual $\boldsymbol{w}^{k-1}$ is equal to the jump of the $(k-1)$-th iterate displacement field across the subdomain interfaces.

- At each FETI PCPG iteration, a matrix-vector product of the form $\boldsymbol{P}^{T} \boldsymbol{r}^{k-1}$ or $\boldsymbol{P} \boldsymbol{z}^{k-1}$ incurs the solution of an auxiliary problem of the form

$$
\left(\boldsymbol{G}_{I}^{T} \boldsymbol{G}_{I}\right) \boldsymbol{x}^{k-1}=\boldsymbol{b}^{k-1}
$$

Hence, the matrix $\boldsymbol{G}_{I}^{T} \boldsymbol{G}_{I}$ (and more generally the matrix $\boldsymbol{G}_{I}^{T} \boldsymbol{Q} \boldsymbol{G}_{I}$ ) defines a coarse problem of size at most equal to $6 N_{s}$, and whose solution couples all the subdomain computations, propagates the error globally, and accelerates convergence. It is because of this coarse problem that for second-order elasticity problems, when the Dirichlet preconditioner is used, the condition number of the FETI interface problem (13) can be bounded by a polylogarithmic function of $H / h$ as follows

$$
\kappa=O\left(1+\log ^{2}\left(\frac{H}{h}\right)\right)
$$


where $H$ and $h$ denote the subdomain and mesh sizes, respectively. The above conditioning result also holds for fourth-order plate and shell problems when the coarse problem (19) is augmented with the so-called subdomain "corner" modes $[14,13,24]$. We remind the reader that a bound such as (20) proves the numerical scalability of the FETI method with respect to both the problem size and the number of subdomains [23, 24] - that is, its ability to solve larger problems using a larger number of subdomains in almost a constant number of iterations. The parallel scalability of the FETI method - that is, its suitability for massively parallel computing - has also been demonstrated on a large number of massively parallel processors for several realistic structural and structural dynamics problems $[17,13]$.

\subsection{Relevance to contact problems}

The subdomains $\Omega^{(s)}$ associated with the FETI method can be viewed as substructures in a state of "perfect" contact. This perfect contact is defined and governed by the compatibility equation (5), which states that the jumps across the substructure interfaces of both the normal and tangential components of the displacement field must vanish. In the FETI method, this perfect contact condition is enforced by discrete Lagrange multipliers whose values are determined by the PCPG algorithm summarized in Table 1 .

The above observations explain our motivation to extend the FETI method to the solution of contact problems whose governing laws are summarized next.

\section{THE FRICTIONLESS CONTACT PROBLEM}

Let $\Omega$ denote the initial state of a linear elastic body, $\partial \Omega_{C}$ the part of its boundary that is a potential area of contact, $n_{C}$ the outward normal to $\partial \Omega_{C}$ at

a point $M, \partial \Omega_{C}^{\prime}$ the potential area of contact with $\partial \Omega_{C}$ located on the surface of a foundation or another linear elastic body, and $n_{C}^{\prime}=-n_{C}$ the outward normal to $\partial \Omega_{C}^{\prime}$ at the point $M^{\prime}$ facing point $M$ (see Figure 2).

If $u$ denotes the displacement field, the discontinuity of $u$ in the normal direction at point $M \in \partial \Omega_{C}$ can be written as

$$
\begin{aligned}
\Delta u(M) & =u(M) \cdot n_{C}+u\left(M^{\prime}\right) \cdot n_{C}^{\prime}=u(M) \cdot n_{C}-u\left(M^{\prime}\right) \cdot n_{C} \\
& =\left(u(M)-u\left(M^{\prime}\right)\right) \cdot n_{C}
\end{aligned}
$$

and the non-penetration condition can be expressed as

$$
\Delta u(M)-c(M)^{0} \leq 0
$$

where $c(M)^{0}$ denotes the initial clearance at point $M$ of the undeformed body $\Omega$ (see Figure 3), and is assumed to be small.

Let $\sigma$ denote the stress tensor, $t=\sigma(M) n$ the traction vector at point $M$, and $t_{n}=t \cdot n$ the normal component of $t$. If $\partial \Omega_{C}$ and $\partial \Omega_{C}^{\prime}$ are in contact, the pressure at $M$ is positive, and therefore $t_{n} \leq 0$ (Figure 4 ). 


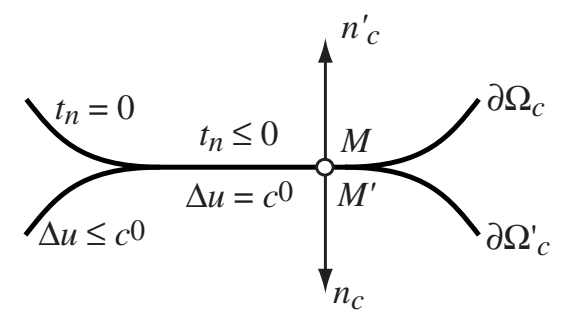

Figure 2: A contact problem

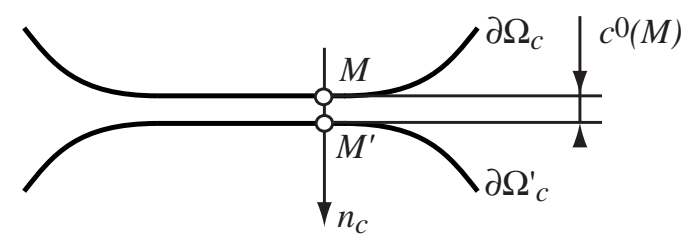

Figure 3: Initial clearance

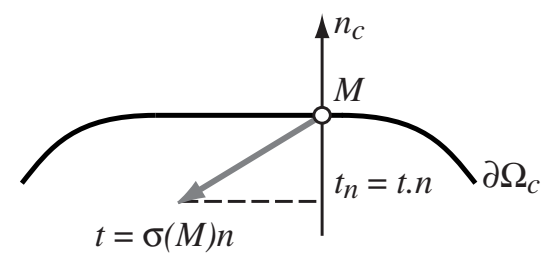

Figure 4: Contact pressure: $t_{n} \leq 0$ 
The contact problem is defined by the unilateral conditions known as the Signorini-Fichera type of conditions

- if $\Delta u(M)-c(M)^{0}=0$ then $t_{n} \leq 0$ and the contact is said to be active.

- if $\Delta u(M)-c(M)^{0}<0$ then $t_{n}=0$ and the contact is said to be inactive.

The above relations are equivalent to stating that on $\partial \Omega_{C}$, the following holds

$$
\begin{aligned}
t_{n} & \leq 0 \\
\Delta u(M)-c(M)^{0} & \leq 0 \\
t_{n} \cdot\left(\Delta u(M)-c(M)^{0}\right) & =0
\end{aligned}
$$

\section{THE FETI-C METHOD}

Our objective is to develop a computationally efficient domain decomposition method for the solution of contact problems that is based on FETI. In this paper, we limit however our attention to frictionless problems. For most domain decomposition methods including FETI, the optimal number of subdomains is dictated by a compromise between several issues related to, among others, computational complexity, memory efficiency, and parallel processing. For this reason, we consider the general case where the number of subdomains $N_{s}$ is larger than the given number of elastic bodies and foundations - that is, the case where each elastic body itself may be decomposed into several subdomains. This leads us to distinguishing between two types of subdomain interfaces: perfect contact interfaces (called perfect interfaces in the sequel), and potential contact interfaces. We construct the FETI-C method as a modified FETI algorithm that (a) distinguishes between perfect and potential contact interfaces, and (b) enforces the compatibility equation (5) on perfect interfaces and the non-penetration condition (22) as well as the Signorini-Fichera conditions (23) on contact interfaces, while (c) satisfying equilibrium and solvability in each subdomain $\Omega^{(s)}$.

\subsection{Preliminaries}

Let

$$
\partial \Omega_{P}^{(s), p}=\partial \Omega^{(s)} \cap \partial \Omega^{(p)}
$$

denote a perfect interface between subdomain $\Omega^{(s)}$ and neighboring subdomain $\Omega^{(p)}$,

$$
\partial \Omega_{C}^{(s), q}=\partial \Omega^{(s)} \cap \partial \Omega^{(q)}
$$

denote a potential contact interface between $\Omega^{(s)}$ and its neighbor $\Omega^{(q)}$,

$$
\partial \Omega_{P}^{(s)}=\bigcup_{p=1}^{p=l} \partial \Omega_{P}^{(s), p}
$$


denote the union of all the perfect interfaces attached to subdomain $\Omega^{(s)}$,

$$
\partial \Omega_{C}^{(s)}=\bigcup_{q=1}^{q=m} \partial \Omega_{C}^{(s), q}
$$

denote the union of all the potential contact interfaces attached to subdomain $\Omega^{(s)}$

$$
\Gamma_{P}=\bigcup_{s=1}^{s=N_{s}} \partial \Omega_{P}^{(s)}
$$

denote the set of all the perfect interfaces associated with the domain decomposition,

$$
\Gamma_{C}=\bigcup_{s=1}^{s=N_{s}} \partial \Omega_{C}^{(s)}
$$

denote the set of all potential contact interfaces and is obtained from the initial configuration of the bodies, and

$$
\Gamma_{I}=\bigcup_{s=1}^{s=N_{s}} \partial \Omega^{(s)}=\Gamma_{P} \cup \Gamma_{C}
$$

denote the global interface associated with the domain decomposition of all given bodies. Using this notation, the edge-wise partition of the interface boundary $\partial \Omega^{(s)}$ of subdomain $\Omega^{(s)}$ can be written as

$$
\begin{aligned}
\partial \Omega^{(s)} & =\partial \Omega_{P}^{(s)} \cup \partial \Omega_{C}^{(s)} \\
& =\left\{\bigcup_{p=1}^{p=l} \partial \Omega_{P}^{(s), p}\right\} \cup\left\{\bigcup_{q=1}^{q=m} \partial \Omega_{C}^{(s), q}\right\}
\end{aligned}
$$

Let $N_{I}$ denote the total number of kinematic equations defined on $\Gamma_{I}$ for the given contact problem, $N_{s, p}$ denote the total number of displacement d.o.f. attached to the perfect interface $\partial \Omega_{P}^{(s), p}$, and $N_{s, q}$ the total number of displacement d.o.f. attached to the potential contact interface $\partial \Omega_{C}^{(s), q}$.

To each perfect interface $\partial \Omega_{P}^{(s), p}$, we associate the matrix $\boldsymbol{b}_{P}^{(s), p} \in \mathcal{R}^{N_{I} \times N_{s, p}}$ defined by

$$
\boldsymbol{b}_{P}^{(s), p} \boldsymbol{v}_{P}^{(s), p}= \begin{cases} \pm \boldsymbol{v}_{P}^{(s), p} & \text { on } \partial \Omega_{P}^{(s), p} \\ 0 & \text { elsewhere on } \Gamma_{I}\end{cases}
$$

where $\boldsymbol{v}^{(s)}$ is a generic vector defined in subdomain $\Omega^{(s)}, \boldsymbol{v}_{P}^{(s), p}$ denotes its trace on the perfect interface $\partial \Omega_{P}^{(s), p}$, and the sign $( \pm)$ is defined by the orientation of the outward normal to $\partial \Omega_{P}^{(s), p}$. Hence, $\boldsymbol{b}_{P}^{(s), p}$ is a signed Boolean matrix. For example, if all the interior d.o.f. of the subdomain $\Omega^{(s)}$ are numbered first, and all the interface boundary d.o.f. of this subdomain are numbered last according 
to the edge-wise partition (32), the signed Boolean matrix $\boldsymbol{B}^{(s)}$ introduced in Section 2.1 for the case without contact can be written as

$$
\boldsymbol{B}^{(s)}=\left[\begin{array}{ll}
\mathbf{0} & \boldsymbol{B}_{I}^{(s)}
\end{array}\right]
$$

where

$$
\boldsymbol{B}_{I}^{(s)}=\left[\begin{array}{lll}
\boldsymbol{b}_{P}^{(s), 1} & \cdots & \boldsymbol{b}_{P}^{(s), l}
\end{array}\right]
$$

To each potential contact interface $\partial \Omega_{C}^{(s), q}$, we associate the matrix $\boldsymbol{b}_{C}^{(s), q} \in$ $\mathcal{R}^{N_{I} \times N_{s, q}}$ defined by

$$
\left(\boldsymbol{b}_{C}^{(s), q} \boldsymbol{v}_{C}^{(s), q}\right)_{M}= \begin{cases}\left(\boldsymbol{n}_{C}^{(s), q^{T}}\right)_{M}\left(\boldsymbol{v}_{C}^{(s), q}\right)_{M} & \text { on } \partial \Omega_{C}^{(s), q} \\ 0 & \text { elsewhere on } \Gamma_{I}\end{cases}
$$

where $\boldsymbol{n}_{C}^{(s), q}$ denotes the vector of outward normals to $\partial \Omega_{C}^{(s), q}$ at each point $M \in \partial \Omega_{C}^{(s), q}$, and the subscript $M$ designates the subvector or entry associated with node $M$. In Eq. (36) and throughout this paper, the subscript $M$ also implies that the stated relation holds at each relevant point $M$. Note that $\boldsymbol{b}_{C}^{(s), q}$ is not a Boolean matrix, except when $\partial \Omega_{C}^{(s), q}$ is parallel to a reference axis.

Hence, for contact problems, we generalize the matrix $\boldsymbol{B}^{(s)}$ introduced in Eq. (3) to account for the zones of potential contact, and therefore transform Eq. (35) into

$$
\boldsymbol{B}_{I}^{(s)}=\left[\begin{array}{lllll}
{\left[\begin{array}{llll}
\boldsymbol{b}_{P}^{(s), 1} & \cdots & \boldsymbol{b}_{P}^{(s), l}
\end{array}\right]} & {\left[\begin{array}{lll}
\boldsymbol{b}_{C}^{(s), 1} & \cdots & \boldsymbol{b}_{C}^{(s), m}
\end{array}\right]}
\end{array}\right]
$$

At each node $M \in \partial \Omega_{C}^{(s), q}$, and for each interface $\partial \Omega_{C}^{(s), q^{\prime}}$ that has the potential of getting in contact with $\partial \Omega_{C}^{(s), q}$, we introduce one potential Lagrange multiplier along the normal direction $n_{C}^{(s), q}$ to $\partial \Omega_{C}^{(s), q}$. Such a Lagrange multiplier represents a contact force in the direction $n_{C}^{(s), q}$.

In the case of contact, the governing subdomain equations $(4,5)$ become

$$
\begin{aligned}
\boldsymbol{K}^{(s)} \boldsymbol{u}^{(s)} & =\boldsymbol{f}^{(s)}-\widetilde{\boldsymbol{B}}^{(s)^{T}} \boldsymbol{\lambda} \quad s=1, \cdots, N_{s} \\
\sum_{s=1}^{N_{s}} \boldsymbol{B}^{(s)} \boldsymbol{u}^{(s)}-\boldsymbol{c}^{0} & \geq \mathbf{0} \text { on } \Gamma_{I}
\end{aligned}
$$

where $\widetilde{\boldsymbol{B}}^{(s)}$ differs from $\boldsymbol{B}^{(s)}$ only when at least one zone of potential contact is inactive (see next section for further details), and $\boldsymbol{c}^{0}$ denotes the extended vector of initial clearances - that is, $c^{0}$ is defined on both $\Gamma_{P}$ and $\Gamma_{C}$ but set to zero on the perfect interfaces. Note that this definition of $c^{0}$ is chosen so that Eq. (39) contain the expected compatibility relation on the perfect interfaces

$$
\sum_{s=1}^{N_{s}} \boldsymbol{B}^{(s)} \boldsymbol{u}^{(s)}=\mathbf{0} \quad \text { on } \Gamma_{P}
$$



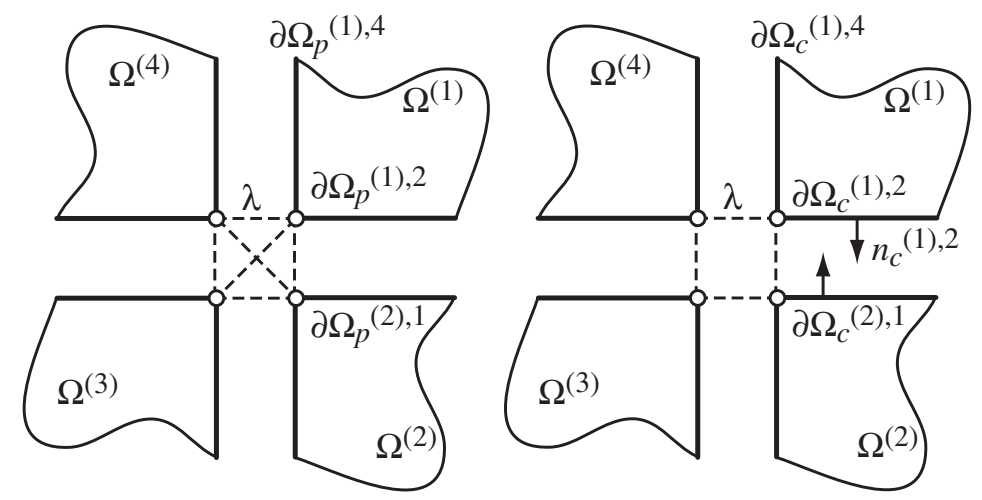

Figure 5: Corner Lagrange multipliers: perfect interfaces (left), contact interfaces (right)

From Eq. (39) and Eq. (38), it follows that for contact problems, the jump $\boldsymbol{w}$ of the displacement field across the subdomain interfaces (see Eq. (18)) is

$$
\boldsymbol{w}=\sum_{s=1}^{N_{s}}\left(\boldsymbol{B}^{(s)} \boldsymbol{K}^{(s)^{+}} \boldsymbol{f}^{(s)}-\boldsymbol{B}^{(s)} \boldsymbol{K}^{(s)^{+}} \widetilde{\boldsymbol{B}}^{(s)^{T}} \boldsymbol{\lambda}\right)+\boldsymbol{G} \boldsymbol{\alpha}-\boldsymbol{c}^{0}
$$

In summary, using the notation introduced in this section, we can re-write the contact conditions (24) on each potential contact interface $\partial \Omega_{C}^{(s), q}$ in terms of FETI variables as follows

$$
\begin{aligned}
\boldsymbol{\lambda}_{M} & \geq 0 \\
\boldsymbol{w}_{M} & \leq 0 \\
\boldsymbol{\lambda}_{M} \boldsymbol{w}_{M} & =0
\end{aligned}
$$

\section{Remark}

- At a crosspoint between four subdomains, six Lagrange multipliers are usually introduced by the standard FETI method to connect together what has been defined in this paper as perfect interfaces. However, from the presentation made above, it follows that at a crosspoint between four subdomains characterized by four potential contact interfaces, only four Lagrange multipliers can be introduced to represent the potential contact forces (Figure 5).

Next, we lay the foundations of the FETI-C method. 


\subsection{Discrete maximization problem}

The standard FETI dual interface problem (8) can be reformulated as follows

$$
\max _{\boldsymbol{\mu} \in \mathcal{R}^{N_{I}}, \boldsymbol{\beta} \in \mathcal{R}^{N_{R}}} \mathcal{C}(\boldsymbol{\mu}, \boldsymbol{\beta})=-\frac{1}{2} \boldsymbol{\mu}^{T} \boldsymbol{F}_{I} \boldsymbol{\mu}+\boldsymbol{\mu}^{T} \boldsymbol{d}+\boldsymbol{\beta}^{T}\left(\boldsymbol{G}_{I}^{T} \boldsymbol{\mu}-\boldsymbol{e}\right)
$$

where $\mathcal{C}(\boldsymbol{\mu}, \boldsymbol{\beta})$ is a complementary energy-like functional, $N_{I}$ has the same meaning as in the previous subsection, and $N_{R}$ is the total number of subdomain rigid body modes. Indeed, differentiating $\mathcal{C}(\boldsymbol{\mu}, \boldsymbol{\beta})$ with respect to $\boldsymbol{\mu}$ and $\boldsymbol{\beta}$ and computing $(\boldsymbol{\lambda}, \boldsymbol{\alpha})$ as the solution of $\left(\frac{\partial \mathcal{C}}{\partial \boldsymbol{\mu}}=0, \frac{\partial \mathcal{C}}{\partial \boldsymbol{\beta}}=0\right)$ leads to the same equations as in (8).

From Eqs. (42), it follows that the standard FETI maximization problem (43) must be modified as follows in order to address contact problems

$$
\max _{\widetilde{\Gamma}_{C}, \boldsymbol{\mu} \in \mathcal{R}^{N_{I}}, \boldsymbol{\mu}_{M} \geq 0, \boldsymbol{\beta} \in \mathcal{R}^{N_{R}}} \mathcal{C}(\boldsymbol{\mu}, \boldsymbol{\beta})=-\frac{1}{2} \boldsymbol{\mu}^{T} \widetilde{\boldsymbol{F}}_{I} \boldsymbol{\mu}+\boldsymbol{\mu}^{T} \boldsymbol{d}+\boldsymbol{\beta}^{T}\left(\widetilde{\boldsymbol{G}}_{I}^{T} \boldsymbol{\mu}-\boldsymbol{e}\right)
$$

where

$$
\begin{aligned}
\widetilde{\boldsymbol{F}}_{I} & =\sum_{s=1}^{N_{s}} \boldsymbol{B}^{(s)} \boldsymbol{K}^{(s)^{+}} \widetilde{\boldsymbol{B}}^{(s)^{T}} \\
\boldsymbol{d} & =\sum_{s=1}^{N_{s}} \boldsymbol{B}^{(s)} \boldsymbol{K}^{(s)^{+}} \boldsymbol{f}^{(s)}-\boldsymbol{c}^{0} \\
\widetilde{\boldsymbol{G}_{I}} & =\left[\begin{array}{lll}
\widetilde{\boldsymbol{B}}^{(1)} \boldsymbol{R}^{(1)} & \cdots & \widetilde{\boldsymbol{B}}^{\left(N_{s}\right)} \boldsymbol{R}^{\left(N_{s}\right)}
\end{array}\right]
\end{aligned}
$$

$\widetilde{\Gamma}_{C}$ is the set of active contact interfaces - that is, the interfaces where $\boldsymbol{\mu}_{M}>0$ - and therefore $\widetilde{\Gamma}_{C}$ verifies

$$
\widetilde{\Gamma}_{C} \subset \Gamma_{C}
$$

$\boldsymbol{B}^{(s)}$ has the same pattern as in Eq. (34), $\boldsymbol{B}_{I}^{(s)}$ is given in Eq. (37),

$$
\begin{gathered}
\widetilde{\boldsymbol{B}}^{(s)}=\left[\begin{array}{cc}
\mathbf{0} & \widetilde{\boldsymbol{B}}_{I}^{(s)}
\end{array}\right] \\
\widetilde{\boldsymbol{B}}_{I}^{(s)}=\left[\begin{array}{lllll}
{\left[\begin{array}{llll}
(s), 1 & \cdots & \boldsymbol{b}_{P}^{(s), l}
\end{array}\right]} & {\left[\begin{array}{lll}
\tilde{\boldsymbol{b}}_{C}^{(s), 1} & \cdots & \tilde{\boldsymbol{b}}_{C}^{(s), m}
\end{array}\right]}
\end{array}\right]
\end{gathered}
$$

and

$$
\left(\tilde{\boldsymbol{b}}_{C}^{(s), q} \boldsymbol{v}_{C}^{(s), q}\right)_{M}= \begin{cases}\left(\boldsymbol{b}_{C}^{(s), q} \boldsymbol{v}_{C}^{(s), q}\right)_{M} & \text { if } M \in \widetilde{\Gamma}_{C} \\ 0 & \text { otherwise }\end{cases}
$$

Let $\tilde{\boldsymbol{w}}$ be defined as follows

$$
\tilde{\boldsymbol{w}}=\left\{\begin{array}{lll}
\boldsymbol{w} & \text { on } & \Gamma_{P} \cup \widetilde{\Gamma}_{C} \\
\left\langle\boldsymbol{w}_{M}\right\rangle_{+} & \text {on } & \Gamma_{C} \backslash \widetilde{\Gamma}_{C}
\end{array}\right.
$$


where $\boldsymbol{w}$ is the jump of the displacement field across the subdomain interfaces as expressed in Eq. (41), and

$$
\langle a\rangle_{+}=\left\{\begin{array}{l}
a \text { if } a>0 \\
0 \text { otherwise }
\end{array}\right.
$$

From the second of Eqs. (42), it follows that if $\boldsymbol{\lambda}$ is solution of the maximization problem (44), and the corresponding subdomain displacement fields $\boldsymbol{u}^{(s)}$ computed from the equilibrium equations (38) satisfy $\tilde{\boldsymbol{w}}=0$, then $\left(\boldsymbol{\lambda},\left\{\boldsymbol{u}^{(s)}\right\}_{s=1}^{s=N_{s}}\right)$ is the domain decomposed solution of the given contact problem.

The FETI-C method we introduce next is a FETI-like method which finds the zone of active contact $\widetilde{\Gamma}_{C}$ and computes the elastic state of each substructure, by solving the maximization problem (44) that embeds the equilibrium equations (38), while ensuring that $\tilde{\boldsymbol{w}}=0$.

\section{Remarks}

- The operator $\widetilde{\boldsymbol{F}}_{I}$ introduced in Eq. (45) is unsymmetric. However, from Eqs. $(37,48,49)$, it follows that the set of admissible Lagrange multipliers on which $\widetilde{\boldsymbol{F}}_{I}$ operates is such that

$$
\widetilde{\boldsymbol{B}}^{T} \boldsymbol{\lambda}=\boldsymbol{B}^{T} \boldsymbol{\lambda}
$$

and therefore a PCG algorithm can be applied to the solution of the maximization problem (44).

- From Eqs. $(9,45)$ and Eq. $(52)$, it also follows that

$$
\widetilde{\boldsymbol{G}}_{I}^{(s)^{T}} \boldsymbol{\lambda}=\boldsymbol{G}_{I}^{(s)^{T}} \boldsymbol{\lambda}
$$

\subsection{One-shot iterative procedure}

As highlighted in the previous sections, the discrete maximization problem (44) associated with contact problems has many similarities with its counterpart (43) for standard structural mechanics problems. For this reason, a good starting point for solving problem (44) is the FETI PCPG algorithm summarized in Table 1. However, a major difference between the two maximization problems (44) and (43) is that the former one is non-linear, whereas the latter one is linear. Another difference pertains to the admissibility of the Lagrange multipliers. For standard structural mechanics problems, the Lagrange multipliers must satisfy the self-equilibrium condition (7) in each floating subdomain. For contact problems, the Lagrange multipliers must also satisfy the additional positivity condition stated in the first of Eqs. (42). Hence, our idea here is to construct a non-linear solution algorithm for solving the discrete maximization problem (44) that mimics the FETI PCPG linear solver, while addressing the differences between perfect and contact interfaces. The resulting non-linear scheme, FETI$\mathrm{C}$, is a FETI-like iterative procedure where both the contact and equilibrium 
conditions are updated simultaneously. Hence, FETI-C does not involve any linearization and therefore unlike the standard FETI solver summarized in Table 1, it cannot by interpreted as a classical PCG algorithm. This new extension of the FETI method is presented in the following subsections in a step-by-step approach.

\subsubsection{Initialization step.}

Consider the FETI-PCPG algorithm summarized in Table 1. Suppose that the initialization step

$$
\boldsymbol{\lambda}^{0}=\boldsymbol{G}_{I}\left(\boldsymbol{G}_{I}^{T} \boldsymbol{G}_{I}\right)^{-1} \boldsymbol{e}
$$

is performed using

$$
\widetilde{\Gamma}_{C}^{0}=\Gamma_{C}
$$

In that case, $\boldsymbol{G}_{I}$ denotes an initialization of $\widetilde{\boldsymbol{G}_{I}}$ (45) where each subdomain matrix $\widetilde{\boldsymbol{B}}_{I}^{(s)}(48)$ is constructed by assuming that all potential contact interfaces are initially active. In other words, $\boldsymbol{G}_{I}^{T} \boldsymbol{G}_{I}$ is based on $\Gamma_{P}$ and $\Gamma_{C}$. If the given problem does not have any global rigid body mode, $\boldsymbol{G}_{I}^{T} \boldsymbol{G}_{I}$ is non-singular [17]. On the other hand, if the given problem has one or several global rigid body modes, $\boldsymbol{G}_{I}^{T} \boldsymbol{G}_{I}$ is singular, but Eq. (54) admits at least one solution if the external loads are self-equilibrated. In any case, the initial value $\boldsymbol{\lambda}^{0}$ as computed in Eq. (54) is not guaranteed to be admissible for the maximization problem (44) - that is, $\boldsymbol{\lambda}^{0}$ is not guaranteed to satisfy $\boldsymbol{\lambda}_{M}^{0}>0$ on $\Gamma_{C}$.

For this reason, we propose to apply after the initialization step a "planing" procedure aimed at enforcing the positiveness of $\boldsymbol{\lambda}^{0}$ in the sense defined in the first of Eqs. (42), without for that purpose destroying the self-equilibrium of any floating subdomain (see Eq. (7)). This planning step is represented by the following transformation

$$
\lambda \longleftarrow \mathcal{P}(\lambda)
$$

where $\mathcal{P}$ denotes the two-step iterative procedure described in Table 2 . In each first step of the $n$-th iteration of this planing procedure, the positiveness of the iterate $\boldsymbol{\lambda}^{n}$ is enforced simply by setting to zero the negative Lagrange multipliers acting on the zone of contact $\Gamma_{C}$, and the active zone of contact $\widetilde{\Gamma}_{C}$ and matrices $\widetilde{\boldsymbol{B}}_{I}^{(s)}$ are updated accordingly and denoted by $\widetilde{\Gamma}_{C}^{n}$ and $\widetilde{\boldsymbol{B}}_{I}^{(s)^{n}}$, respectively. In each second step, the self-equilibrium of the floating subdomains is restored by applying to $\boldsymbol{\lambda}^{n}$ the following projector

$$
\widetilde{\boldsymbol{P}}^{n}=\boldsymbol{I}-\widetilde{\boldsymbol{G}}_{I}^{n}\left(\widetilde{\boldsymbol{G}}_{I}^{n^{T}} \widetilde{\boldsymbol{G}}_{I}^{n}\right)^{-1} \boldsymbol{G}_{I}^{n^{T}}
$$

and translating the result by the constant vector $\widetilde{\boldsymbol{G}}_{I}^{n}\left(\widetilde{\boldsymbol{G}}_{I}^{n^{T}} \widetilde{\boldsymbol{G}}_{I}^{n}\right)^{-1} \boldsymbol{e}$. Indeed, from Eq. (57) and Eq. (53), it follows that

$$
\widetilde{\boldsymbol{G}}_{I}^{n^{T}}\left(\widetilde{\boldsymbol{P}}^{n} \boldsymbol{\lambda}^{n}+\widetilde{\boldsymbol{G}}_{I}^{n}\left(\widetilde{\boldsymbol{G}}_{I}^{n^{T}} \widetilde{\boldsymbol{G}}_{I}^{n}\right)^{-1} \boldsymbol{e}\right)=\left(\widetilde{\boldsymbol{G}}_{I}^{n^{T}}-\boldsymbol{G}_{I}^{n^{T}}\right) \boldsymbol{\lambda}^{n}+\boldsymbol{e}=\boldsymbol{e}
$$

Note that the projector (57) is similar to the projector (12) equipped with $\boldsymbol{Q}=\boldsymbol{I}$, but differs from it in the fact that it accounts for the change in the 
Table 2: The planing procedure

\begin{tabular}{ll}
\hline Iterate $n=1,2, \cdots$ & until convergence \\
\hline 1.(a) Positiveness: & $\boldsymbol{\lambda}^{n} \longleftarrow \begin{cases}\boldsymbol{\lambda}^{n} & \text { on } \Gamma_{P} \\
\left\langle\boldsymbol{\lambda}_{M}^{n}\right\rangle_{+} & \text {on } \Gamma_{C}\end{cases}$ \\
1.(b) Active contact: & determine $\widetilde{\Gamma}_{C}^{n}$ from $\boldsymbol{\lambda}^{n}$ and update $\widetilde{\boldsymbol{B}}_{I}^{(s)^{n}}, \widetilde{\boldsymbol{G}}_{I}{ }^{n}$ \\
1.(c) Test self-equilibrium: & if $\widetilde{\boldsymbol{G}}_{I}{ }^{T} \boldsymbol{\lambda}^{n}=\boldsymbol{e}$ end. \\
2.(a) Self-equilibrium: & $\boldsymbol{\lambda}^{n} \longleftarrow\left(\boldsymbol{I}-\widetilde{\boldsymbol{G}}_{I}^{n}\left(\widetilde{\boldsymbol{G}}_{I}{ }^{T} \widetilde{\boldsymbol{G}}_{I}{ }^{n}\right)^{-1} \boldsymbol{G}_{I}^{n^{T}}\right) \boldsymbol{\lambda}^{n}+\widetilde{\boldsymbol{G}}_{I}^{n}\left(\widetilde{\boldsymbol{G}}_{I}^{n^{T}} \widetilde{\boldsymbol{G}}_{I}{ }^{n}\right)^{-1} \boldsymbol{e}$ \\
2.(b) Test positiveness: & if $\exists M \in \Gamma_{C} / \boldsymbol{\lambda}_{M}^{n}<0$ go to $1 .(\mathrm{a})$ \\
\hline
\end{tabular}

active zone of contact from $\widetilde{\Gamma}_{C}^{n-1}$ to $\widetilde{\Gamma}_{C}^{n}$. Experience shows that in general, the planing procedure described in Table 2 converges after a small number of planing subiterations (see Section 5).

Note also that Step 2.(a) of the planing procedure incorporates a coarse problem of the form

$$
\left(\widetilde{\boldsymbol{G}}_{I}^{n^{T}} \widetilde{\boldsymbol{G}}_{I}^{n}\right) \boldsymbol{x}^{n}=\boldsymbol{b}^{n}
$$

This coarse problem is called here a coarse "contact" problem because, as shown in Table 2, it influences the updating of the active zone of contact.

\subsubsection{Evaluation of the projected residual.}

As noted in Section 4.2, for contact problems, it is the modified projected residual $\tilde{\boldsymbol{w}}$ defined in Eq. (50) and not the projected residual $\boldsymbol{w}(18)$ that must be driven to zero. For this reason, at each iteration $k$, after $\boldsymbol{w}^{k-1}=\widetilde{\boldsymbol{P}}^{k-1^{T}} \boldsymbol{r}^{k-1}$ is evaluated, $\tilde{\boldsymbol{w}}^{k-1}$ is computed and the preconditioner is applied to $\tilde{\boldsymbol{w}}^{k-1}$ rather than $\boldsymbol{w}^{k-1}$. For the same reason, $\tilde{\boldsymbol{w}}^{k-1}$ and $\tilde{\boldsymbol{w}}^{k-2}$ are used in FETI-C wherever $\boldsymbol{w}^{k-1}$ and $\boldsymbol{w}^{k-2}$ are used in FETI.

The re-projection step is similar to that of the standard FETI PCPG solver and can be written as $\boldsymbol{y}^{k-1}=\widetilde{\boldsymbol{P}}^{k-1} \boldsymbol{z}^{k-1}$, where $\boldsymbol{z}^{k-1}=\widetilde{\boldsymbol{F}}_{I}^{-1} \tilde{\boldsymbol{w}}^{k-1}$, and $\overline{\boldsymbol{F}}_{I}^{-1}$ is the preconditioner discussed next.

\subsubsection{Preconditioning step.}

The straightforward extension to contact problems of the Dirichlet (15) preconditioner calls for substituting the matrices $\boldsymbol{B}^{(s)}$ in Eqs. $(15,16)$ by the matrices $\widetilde{\boldsymbol{B}}^{(s)^{k}}$ associated with the $k$-th iterate active zone of contact $\widetilde{\Gamma}_{C}^{k}$, and updating the definition of the interior (subscript $i$ ) and interface boundary (subscript $b$ ) d.o.f. accordingly. However, in order to avoid non-desirable oscillations in the enforcement of the non-penetration condition (second of Eqs. (42)), we propose to precondition only the equations corresponding to the d.o.f. where the modified projected residual $\tilde{\boldsymbol{w}}$ is non-zero. Hence, we propose the following 
"Dirichlet-like" preconditioner

$$
\overline{\widetilde{\boldsymbol{F}}}_{I}^{D^{-1 k}}=\boldsymbol{W}\left(\sum_{s=1}^{N_{s}} \widetilde{\widetilde{\boldsymbol{B}}}^{(s)^{k}}\left[\begin{array}{cc}
\mathbf{0} & \mathbf{0} \\
\mathbf{0} & \boldsymbol{S}_{b b}^{(s)}
\end{array}\right] \widetilde{\widetilde{\boldsymbol{B}}}^{(s)^{k T}}\right) \boldsymbol{W}
$$

where $\widetilde{\boldsymbol{B}}^{(s)^{k}}$ is the restriction of $\boldsymbol{B}^{(s)^{k}}$ to the d.o.f. of $\Gamma_{P}$ and to the d.o.f. of $\Gamma_{C}^{k}$ where the effective residual $\tilde{\boldsymbol{w}}^{k-1}$ is non-zero. For simplicity, we refer in the sequel to the Dirichlet-like preconditioner introduced above as the Dirichlet preconditioner.

At each iteration $k$, the above Dirichlet preconditioner changes because the d.o.f. on $\Gamma_{C}$ where $\tilde{\boldsymbol{w}}^{k-1} \neq 0$ change. However, only the matrices $\widetilde{\widetilde{\boldsymbol{B}}}^{(s)}$ are updated. The Schur complement $\boldsymbol{S}_{b b}^{(s)}$ is computed once based on the initial configuration - that is, on $\Gamma_{P} \cup \widetilde{\Gamma}_{C}^{0}=\Gamma_{P} \cup \Gamma_{C}$ - and is frozen throughout all iterations. This strategy is chosen to maximize computational efficiency.

Note that the fact that the above preconditioner changes at each iteration $k$ is a direct consequence of the choice we have made to design a non-linear FETI$\mathrm{C}$ solution algorithm that does not require any linearization of the non-linear maximization problem (44).

\subsubsection{Conjugation and maximization steps.}

The derivation and explanation of the conjugation and maximization (or minimization) steps that characterize a PCG algorithm are usually performed for a linear problem. In general, these steps are not required for a non-linear problem. Nevertheless, we enforce them in the FETI-C solver in order to avoid as much as possible numerical oscillations. However, in this case and even in the absence of round-off errors, the conjugation step enforces the orthogonality of the current search direction only to the previous one.

\subsubsection{Planing step.}

At each iteration $k$, the planing procedure (56) (or planing step) is applied to the updated Lagrange multipliers in order to enforce both the positiveness of the contact pressure and the self-equilibrium of the floating subdomains. Note that at the end of each $k$-th application of the planing procedure, a possibly new active zone of contact $\widetilde{\Gamma}_{C}^{k+1}$ is predicted, and therefore is available for updating the matrices $\widetilde{\boldsymbol{B}}^{(s)}$ to become $\widetilde{\boldsymbol{B}}^{()^{k}}$, and the projector $\widetilde{\boldsymbol{P}}$ and the precondititioner $\overline{\widetilde{\boldsymbol{F}}}_{I}^{-1}$ to become $\widetilde{\boldsymbol{P}}^{k}$ and $\overline{\widetilde{\boldsymbol{F}}}_{I}^{-1^{k+1}}$, respectively.

\subsubsection{Evaluation of the residual.}

In the sequel, we define a "status change" as the event where any node on the potential zone of contact $\Gamma_{C}$ changes status from active to inactive, or viceversa, during a FETI-C iteration. A status change affects the evaluation of the residual. 
Indeed, we first note that the residual can be computed only after the planing step, and that after each planing step the predicted active zone of contact is updated. If a status change occurs during this update, $\widetilde{\boldsymbol{F}}_{I}$ is modified, which prevents evaluating the residual as in a classical CG algorithm. Instead, if a status change occurs, this residual but must be re-computed as follows

$$
\boldsymbol{r}^{k}=\boldsymbol{d}-\widetilde{\boldsymbol{F}}_{I}^{k+1} \boldsymbol{\lambda}^{k}
$$

where the notation $\widetilde{\boldsymbol{F}}_{I}^{k+1}$ means $\widetilde{\boldsymbol{F}}_{I}^{k+1}=\widetilde{\boldsymbol{F}}_{I}\left(\widetilde{\Gamma}_{C}^{k+1}\right)$ and emphasizes the fact that $\widetilde{\boldsymbol{F}}_{I}$ depends on the predicted active zone of contact $\widetilde{\Gamma}_{C}$ via the matrices $\widetilde{\boldsymbol{B}}^{(s)}$ (see Eqs. (45-49)). On the other hand, if no status change occurs during the updating of the active zone of contact, the residual can be computed as in the classical CG algorithm.

\subsection{The FETI-C solver}

The FETI-C solver for frictionless contact problems is summarized in Table 3. Convergence is checked by monitoring $\tilde{\boldsymbol{w}}^{k-1}$ and declared when

$$
\left\|\tilde{\boldsymbol{w}}^{k-1}\right\| \leq \epsilon\left(\left\|\sum_{s=1}^{N_{s}} \boldsymbol{B}^{(s)} \boldsymbol{K}^{(s)^{+}} \boldsymbol{f}^{(s)}\right\|+\left\|\boldsymbol{c}^{0}\right\|\right)
$$

where $\epsilon$ is a user-specified tolerance, and the triangular inequality has been applied to $\|\boldsymbol{d}\|(45)$ to avoid potential numerical difficulties.

\section{NUMERICAL EXAMPLES}

In this section, we assess the performance of the FETI-C solver and investigate its numerical scalability properties. For this purpose, we apply this algorithm, with and without preconditioning, to the solution of several two- and threedimensional frictionless contact problems. In all cases we monitor its convergence as described in (62) with $\epsilon=10^{-7}$.

Furthermore, we use the performance of the optimal FETI method as a reference to evaluate the numerical efficiency of the FETI-C solver, in the following manner. For each contact problem, after the FETI-C solver has converged and the active zone of contact has been determined, we "lock" the substructures in their final configuration - that is, we consider each determined active contact interface as a perfect interface in the normal direction - and perform a standard stress analysis of that configuration under the given external loads, using the standard FETI method as a solver. We compare the dependence on a few key parameters of the number of FETI iterations associated with this stress analysis to that of the number of FETI-C iterations performed during the solution of the underlying contact problem. 
Table 3: The FETI-C non-linear solution algorithm

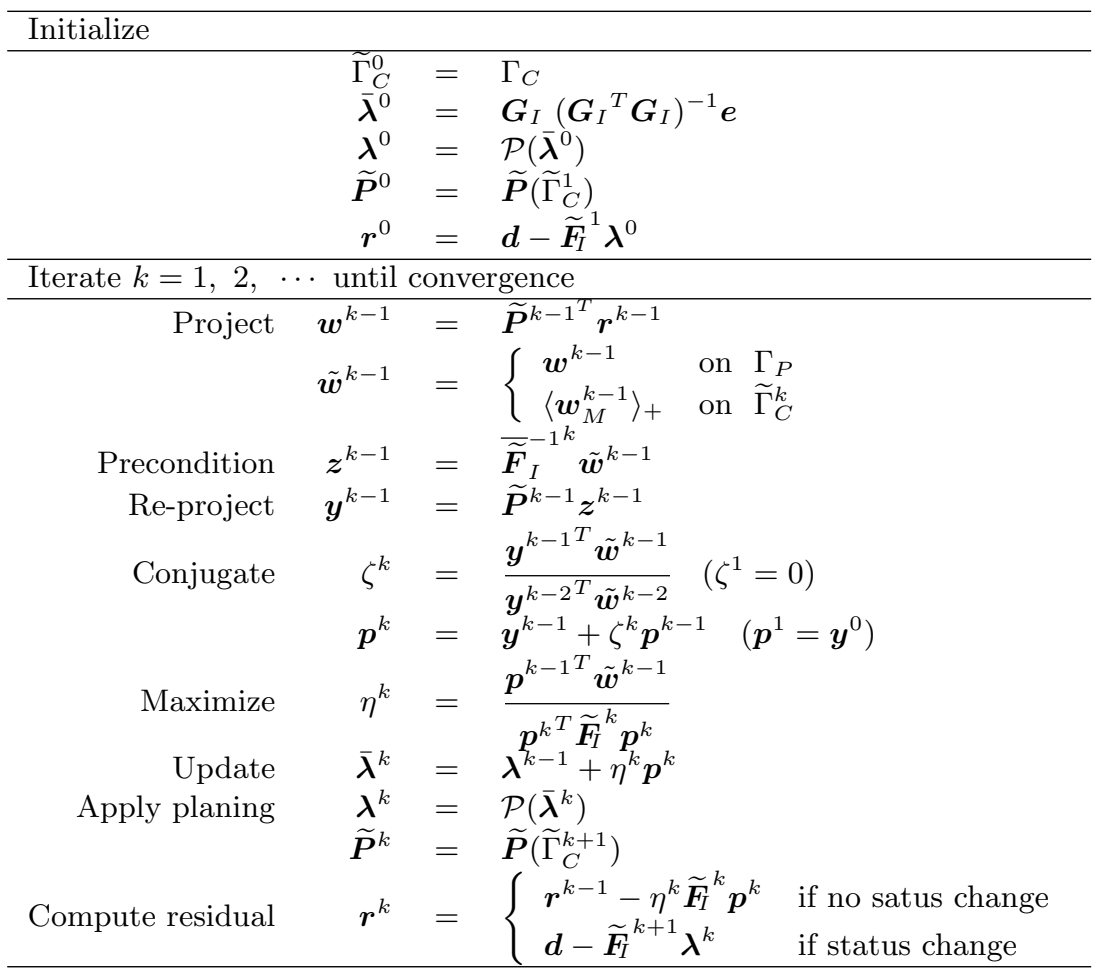




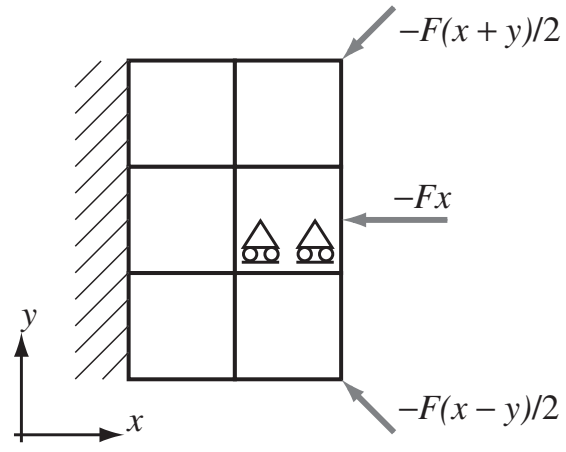

Figure 6: A 6-block problem with 7 contact interfaces

\subsection{Two-dimensional applications}

Here, we consider a series of two-dimensional contact problems between 2 to 6 substructures. Each one of this problem features a different challenging aspect of contact. We assume that all substructures are made of the same linear elastic material characterized by a Young modulus $E=2.0510^{3} \mathrm{MPa}$, and a Poisson ratio $\nu=0.3$. We discretize all substructures by four-noded plane stress elements, design small meshes with 1,364 d.o.f. as well as larger ones with up to 175,692 d.o.f., and vary the total number of subdomains between 6 and 150 .

\subsubsection{A multi-block problem.}

First, we consider the problem graphically depicted in Figure 6, with $F=10^{4} \mathrm{~N}$. In this problem, 6 blocks sharing 7 contact interfaces are pushed against each other by a set of external forces. The two simple supports drawn in one of the blocks mean that the interface boundary nodes of that block can move only in the horizontal $(x)$ direction. This condition ensures that the contact problem is well-posed and has a unique solution.

We construct three different domain decompositions with $6(H=1 / 2), 54$ $(H=1 / 6)$, and $150(H=1 / 10)$ subdomains (Figure 7$)$. In each subdomain, we generate a suite of $(1 / h) \times(1 / h)$ uniform meshes with $10 \leq(H / h) \leq 60$. For each domain decomposition and each mesh size, we report in Table 4 the performance results of the FETI-C solver. These consist of the number of FETIlike iterations $N_{i t r}$, the total number of planing subiterations $N_{p l n}$, and the total number of status changes $N_{s t c}$. We denote by $N_{d o f}$ the total number of d.o.f. associated with a given mesh, and by $N_{i t r}^{\text {lock }}$ the number of FETI iterations obtained for the solution of the locked configuration of this contact problem with Dirichlet preconditioner.

The performance results summarized in Table 4 show that

- The Dirichlet preconditioner reduces the number of FETI-C iterations by a factor ranging between 2 and 3 . 


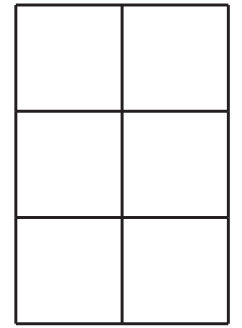

6 subdomains

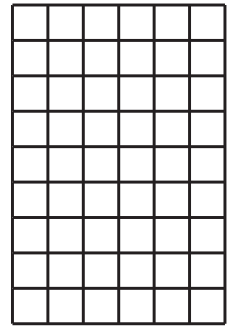

54 subdomains

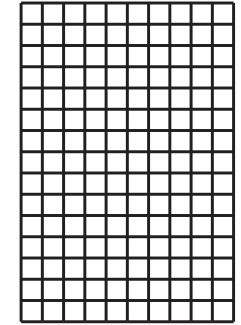

150 subdomains

Figure 7: Domain decomposition

- The FETI-C solver equipped with the Dirichlet preconditioner is numerically scalable with respect to both the problem size and the number of subdomains.

- For this contact problem, the FETI-C solver equipped with the Dirichlet preconditioner performs an amazingly small number of planing subiterations. Furthermore, for large-scale meshes and mesh partitions, it requires a number of iterations that is comparable to that of the FETI method applied to the standard stress analysis of the locked configuration of this problem.

The third observation formulated above can be explained by Figure 8 where the displacement solution of this contact problem using the coarsest mesh is displayed on the left, and the distribution of the contact Lagrange multipliers is graphically depicted using arrows on the right. In this figure, the reader can observe that almost all contact interfaces are active, which explains why few planing subiterations are required and $N_{i t r}$ is close to $N_{i t r}^{\text {lock }}$. The deformed shape shown on the left in Figure 8 also illustrates the reason why, at a crosspoint, no Lagrange multiplier should be introduced between two subdomains that share only one node in two-dimensions, or on edge in three-dimensions (see the remark in Section 4.1).

\subsubsection{A contact problem with pre-stress.}

Next, we consider a frictionless contact problem where a square elastic body is forced into the cavity of another U-shaped elastic body, as shown in Figure 9 with $c^{0}=-10^{-6} \mathrm{~m}$ and $F=100 \mathrm{~N}$. This problem has only 3 potential contact interfaces. However, it has the same topology as the previous problem. For this reason, we use the same meshes and same domain decompositions as for the previous problem (see Figure 7). We report in Table 5 and Figure 10 the performance results obtained for the FETI-C solver.

The reported values of $N_{i t r}^{\text {lock }}$ suggest that, even for a standard linear configuration, this problem is intrinsically "harder" to solve by a FETI method 
Table 4: Multi-block contact problem: performance results of the FETI-C solver

\begin{tabular}{cccccccc}
\hline$H\left(N_{s}\right)$ & $H / h$ & $N_{\text {dof }}$ & preconditioner & $N_{\text {itr }}$ & $N_{\text {pln }}$ & $N_{\text {stc }}$ & $N_{\text {itr }}^{\text {lock }}$ \\
\hline $1 / 2(6)$ & 10 & 1,452 & $\boldsymbol{I}$ & 23 & 0 & 0 & \\
$1 / 2(6)$ & 20 & 5,292 & $\boldsymbol{I}$ & 31 & 2 & 2 & \\
$1 / 2(6)$ & 40 & 19,200 & $\boldsymbol{I}$ & 43 & 4 & 3 & \\
$1 / 2(6)$ & 60 & 44,652 & $\boldsymbol{I}$ & 50 & 4 & 3 & \\
$1 / 6(54)$ & 10 & 11,532 & $\boldsymbol{I}$ & 52 & 3 & 2 & \\
$1 / 6(54)$ & 20 & 44,652 & $\boldsymbol{I}$ & 68 & 6 & 3 & \\
$1 / 6(54)$ & 40 & 175,692 & $\boldsymbol{I}$ & 88 & 11 & 5 & \\
$1 / 10(150)$ & 10 & 31,212 & $\boldsymbol{I}$ & 57 & 8 & 5 & \\
\hline $1 / 2(6)$ & 10 & 1,452 & Dirichlet & 8 & 0 & 0 & 7 \\
$1 / 2(6)$ & 20 & 5,292 & Dirichlet & 11 & 0 & 0 & 7 \\
$1 / 2(6)$ & 40 & 19,200 & Dirichlet & 13 & 0 & 0 & 6 \\
$1 / 2(6)$ & 60 & 44,652 & Dirichlet & 13 & 1 & 1 & 7 \\
$1 / 6(54)$ & 10 & 11,532 & Dirichlet & 30 & 0 & 0 & 24 \\
$1 / 6(54)$ & 20 & 44,652 & Dirichlet & 34 & 0 & 0 & 26 \\
$1 / 6(54)$ & 40 & 175,692 & Dirichlet & 36 & 3 & 1 & 29 \\
$1 / 10(150)$ & 10 & 31,212 & Dirichlet & 34 & 5 & 3 & 30 \\
\hline
\end{tabular}
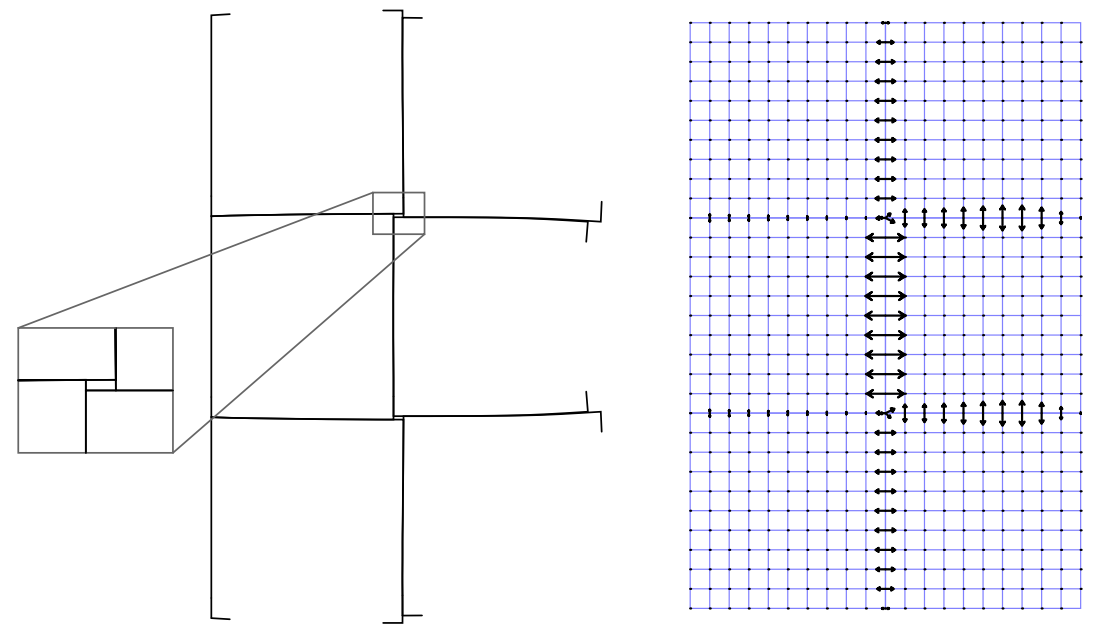

Figure 8: Multi-block contact problem: deformed interfaces (left) and corresponding Lagrange multipliers (right, arrows) 


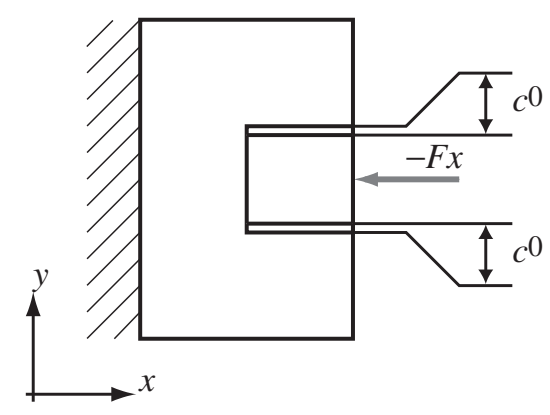

Figure 9: A contact problem with a pre-stressed block

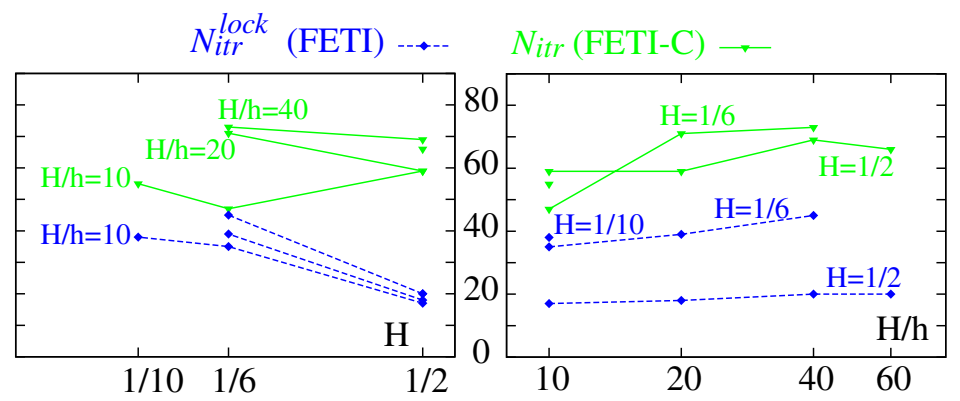

Figure 10: Contact problem with a pre-stressed block: performance results of the FETI-C solver equipped with the Dirichlet preconditioner

than the previous one. This explains why the FETI-C method performs more iterations for solving this problem than for solving the previous one. Nevertheless, the performance results summarized in Table 5 also show that the FETI-C solver equipped with the Dirichlet preconditioner: (a) is numerically scalable with respect to both the problem size and the number of subdomains, and (b) performs on average only 1 or 2 planing subiterations per iteration. The latter observation suggests that the coarse contact problem (59) is effective at reducing the oscillations that are usually observed when determining the active contact zone by an iterative procedure. Figure 11 reports on the evolution of the relative projected residual (62), number of planing subiterations, and number of nodes that experience a status change throughout the iterations, for the case with 54 subdomains, $H / h=40$, and the Dirichlet preconditioner. The reader can observe that after a few iterations, no status change occurs and therefore the evaluation of the residual becomes as economical as in a classical CG algorithm.

The solution of this contact problem with pre-stress is graphically depicted in Figure 12. The gap between the deformed interfaces of the two elastic bodies is due to the fact that the binding $c^{0}$ has been prescribed with respect to the 
Table 5: Contact problem with a pre-stressed block: performance results of the FETI-C solver

\begin{tabular}{cccccccc}
\hline$H\left(N_{s}\right)$ & $H / h$ & $N_{\text {dof }}$ & preconditioner & $N_{\text {itr }}$ & $N_{\text {pln }}$ & $N_{\text {stc }}$ & $N_{i t r}^{\text {lock }}$ \\
\hline $1 / 2(6)$ & 10 & 1,364 & $\boldsymbol{I}$ & 53 & 21 & 7 & \\
$1 / 2(6)$ & 20 & 5,124 & $\boldsymbol{I}$ & 62 & 43 & 9 & \\
$1 / 2(6)$ & 40 & 19,844 & $\boldsymbol{I}$ & 84 & 32 & 11 & \\
$1 / 2(6)$ & 60 & 44,164 & $\boldsymbol{I}$ & 105 & 43 & 17 & \\
$1 / 6(54)$ & 10 & 11,284 & $\boldsymbol{I}$ & 63 & 14 & 4 & \\
$1 / 6(54)$ & 20 & 44,164 & $\boldsymbol{I}$ & 89 & 58 & 11 & \\
$1 / 6(54)$ & 40 & 174,724 & $\boldsymbol{I}$ & 121 & 80 & 14 & \\
$1 / 10(150)$ & 10 & 30,804 & $\boldsymbol{I}$ & 68 & 29 & 8 & \\
\hline $1 / 2(6)$ & 10 & 1,364 & Dirichlet & 59 & 21 & 7 & 17 \\
$1 / 2(6)$ & 20 & 5,124 & Dirichlet & 59 & 38 & 6 & 18 \\
$1 / 2(6)$ & 40 & 19,844 & Dirichlet & 69 & 49 & 12 & 20 \\
$1 / 2(6)$ & 60 & 44,164 & Dirichlet & 66 & 55 & 11 & 20 \\
$1 / 6(54)$ & 10 & 11,284 & Dirichlet & 47 & 16 & 7 & 35 \\
$1 / 6(54)$ & 20 & 44,164 & Dirichlet & 71 & 41 & 10 & 39 \\
$1 / 6(54)$ & 40 & 174,724 & Dirichlet & 73 & 37 & 13 & 45 \\
$1 / 10(150)$ & 10 & 30,804 & Dirichlet & 55 & 40 & 6 & 38 \\
\hline
\end{tabular}
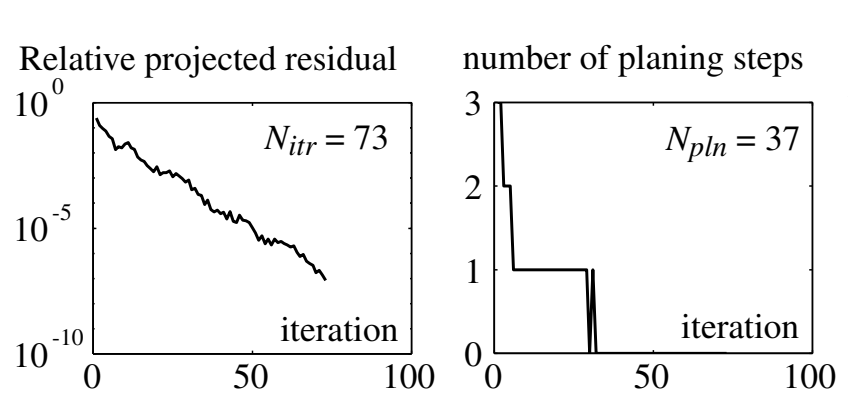

number of nodes experiencing a status change

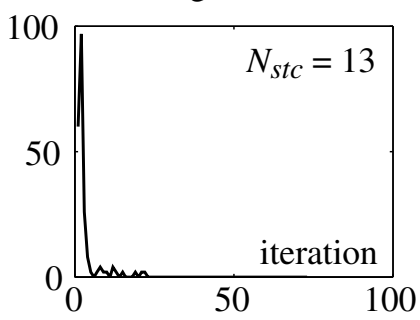

Figure 11: Convergence behavior of the FETI-C solver for the contact problem with a pre-stressed block 

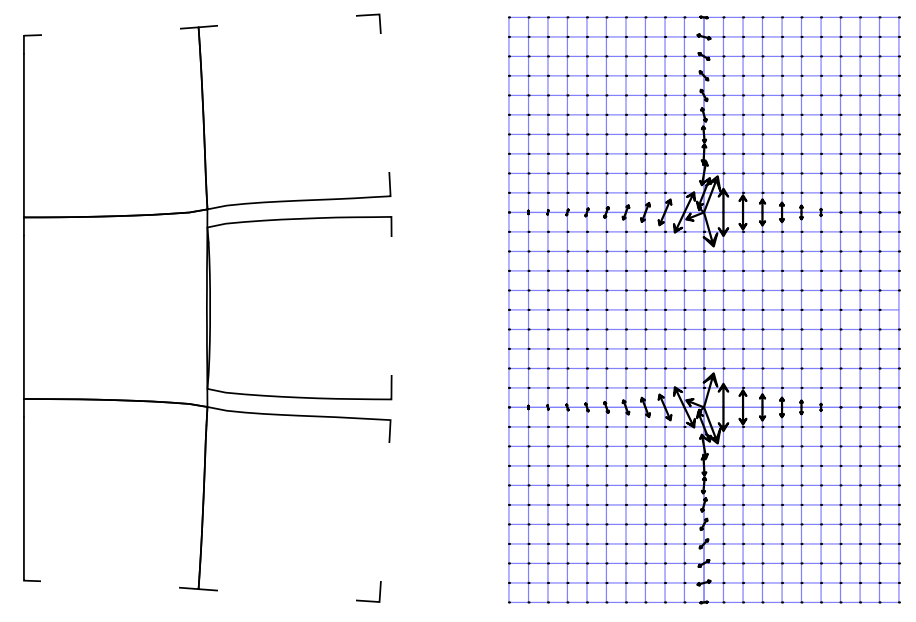

Figure 12: Contact problem with a pre-stressed block: deformed interfaces (left) and corresponding Lagrange multipliers (right, arrows)

undeformed configuration.

\subsection{A three-dimensional contact problem}

In order to test the behavior of the planing procedure (56) on two-dimensional interfaces, we finally consider the three-dimensional frictionless contact problem shown in Figure 13. In this problem - which is a three-dimensional version of the contact problem described in Section 5.1.1 - the translation in the $z$ direction is restrained for all of substructures 4,5 , and 6 , and the translation in the $y$ direction as well as the rotation around the $x$ axis are restrained for substructure 5. The loading is identical to that of the multi-block problem shown in Figure 6 , but only $80 \%$ of the potential contact nodes turn out to be active in this problem.

We generate two different meshes corresponding to discretizing each substructure by $4 \times 4 \times 4$ and $12 \times 12 \times 128$-noded brick elements. Hence, the first mesh contains 2,250 d.o.f., and the second one 39, 546 d.o.f. We treat each substructure as one subdomain. We report in Table 6 the performance results of the FETI-C solver applied to this contact problem. Once again, these results show that the FETI-C solver equipped with the Dirichlet preconditioner performs on average about 2 planing subiterations per iteration, and is scalable with respect to both the size of the problem and the number of subdomains.

\subsection{On the solution of the coarse contact problem}

The implementation on sequential and parallel computing platforms of the FETI-C solver presented in this paper is similar to that of the FETI method. 


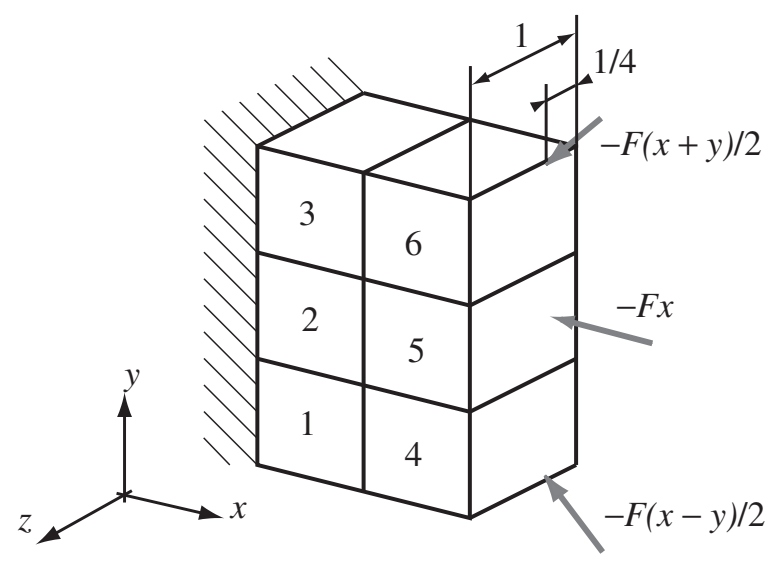

Figure 13: A three-dimensional 6-block contact problem

Table 6: Three-dimensional 6-block contact problem: performance results of the FETI-C solver

\begin{tabular}{cccccccc}
\hline$H\left(N_{s}\right)$ & $H / h$ & $N_{\text {dof }}$ & preconditioner & $N_{\text {itr }}$ & $N_{\text {pln }}$ & $N_{\text {stc }}$ & $N_{\text {itr }}^{\text {lock }}$ \\
\hline $1 / 2(6)$ & 4 & 2,250 & $\boldsymbol{I}$ & 18 & 11 & 3 & \\
$1 / 2(6)$ & 12 & 39,546 & $\boldsymbol{I}$ & 15 & 18 & 13 & \\
\hline $1 / 2(6)$ & 4 & 2,250 & Dirichlet & 11 & 6 & 3 & 6 \\
$1 / 2(6)$ & 12 & 39,546 & Dirichlet & 8 & 17 & 7 & 5 \\
\hline
\end{tabular}


It will be discussed in details in a forthcoming paper, which will also report on CPU performance results. Here, we make only one comment on the solution of the coarse contact problem (59). When the number of potential contact interfaces can be expected to be a small fraction of the total number of interfaces - for example, when only a few bodies are in contact and a large number of subdomains is employed - we recommend that the contribution of the perfect interfaces to $\left(\widetilde{\boldsymbol{G}}_{I}^{n^{T}} \widetilde{\boldsymbol{G}}_{I}^{n}\right)$ be eliminated once for all by a static condensation procedure, before starting the FETI-C iterations. In that case, at each planing subiteration of a given FETI-C iteration, the cost of solving the coarse contact problem (59) becomes negligible compared to the other computational costs of the FETI-C method.

\section{SUMMARY AND CONCLUSIONS}

The FETI-C method is a FETI-based substructuring method with Lagrange multipliers aimed for the iterative solution of general contact problems. As a first step, it is developed in this paper for frictionless problems. It is organized around a single iteration loop that updates both the contact and equilibrium conditions. A distinctive feature of the FETI-C method is an iterative planing procedure which enforces at each FETI-C iteration the positiveness of the contact pressure while preserving the self-equilibrium of each floating subdomain. This planing procedure incorporates an auxiliary coarse problem which not only guides the prediction of the active zone of contact, but most importantly appears to be effective at reducing the oscillations that are usually observed when determining the active contact zone by an iterative procedure. The computational cost of each planing subiteration is a minor fraction of the computational cost of a FETI-C iteration. Furthermore, extensive numerical experiments reveal that the FETI-C method performs on average less than 2 planing subiterations per iteration. Extensive numerical experiments with two- and three-dimensional frictionles contact problems also suggest that the FETI-C method is numerically scalable with respect to both the number of subdomains, and the size of the problem. The extension of this method to contact problems with friction necessitates (a) modifying the admissibility condition on the Lagrange multipliers introduced at the contact interfaces to account for friction, and (b) superposing to the current residuals additional terms that account for the tangential components of the jumps of the displacement field. These issues will be dealt with in a forthcoming paper.

Acknowledgements The first author acknowledges partial support by the Service des Relations Internationales of the ENS Cachan. The second author acknowledges the support by the Sandia National Laboratories under Contract No. BD-2435. 


\section{References}

[1] P. Alart and A. Curnier. A mixed formulation for frictional contact problems prone to Newton like solution methods. Computer Methods in Applied Mechanics and Engineering, 92:253-375, 1991.

[2] F. Armero and E. Petöcz. Formulation and analysis of conserving algorithms for dynamic contact / impact problems. Computer Methods in Applied Mechanics and Engineering, (158):269-300, 1998.

[3] J. S. Arora, A. I. Chahande, and J. K. Paeng. Multiplier methods for engineering optimization. International Journal for Numerical Methods in Engineering, 32:1485-1525, 1991.

[4] K. J. Arrow, L. Hurwicz, and H. Uzawa. Studies in nonlinear programming. University Press, Stanford, CA, 1958.

[5] M. Barboteu, P. Alart, and F. Lebon. Une méthode de décomposition de domaine pour une structure multicontact. In 4 e Colloque National en Calcul des Structures, volume 1, pages 159-164, 1999.

[6] P. Chabrand, F. Dubois, and M. Raous. Various numerical methods for solving unilateral contact problems with friction. In Rodin and Shillor [30], pages $97-108$.

[7] L. Champaney, J.-Y. Cognard, D. Dureisseix, and P. Ladevèze. Large scale applications on parallel computers of a mixed domain decomposition method. Computational Mechanics, (19):253-263, 1997.

[8] L. Champaney, J.-Y. Cognard, and P. Ladevèze. Modular analysis of assemblages of three-dimensional structures with unilateral contact conditions. Computers \&5 Structures, (73):249-266, 1999.

[9] A. Curnier, editor. Proceedings of the Contact Mechanics International Symposium. Presses Polytechniques et Universitaires Romandes, 1992.

[10] G. Dilintas, P. Laurent-Gengoux, and D. Trystram. A conjugate projected gradient method with preconditioning for unilateral contact problems. Computers \&3 Structures, 29(4):675-680, 1988.

[11] Z. Dostal, A. Friedlander, and S. Santos. Solution of coercive and semicoercive contact problems by FETI domain decomposition. Contemporary Mathematics, 218:82-93, 1998.

[12] C. Farhat. A Lagrange multiplier based divide and conquer finite element algorithm. Journal of Computing Systems in Engineering, 2:149-156, 1991.

[13] C. Farhat, P. S. Chen, J. Mandel, and F. X. Roux. The two-level FETI method - Part II: extension to shell problems, parallel implementation and performance results. Computer Methods in Applied Mechanics and Engineering, 155:153-180, 1998. 
[14] C. Farhat and J. Mandel. The two-level FETI method for static and dynamic plate problems - Part I: an optimal iterative solver for biharmonic systems. Computer Methods in Applied Mechanics and Engineering, 155:129-152, 1998.

[15] C. Farhat, J. Mandel, and F. X. Roux. Optimal convergence properties of the FETI domain decomposition method. Computer Methods in Applied Mechanics and Engineering, 115:367-388, 1994.

[16] C. Farhat and F.-X. Roux. A method of finite element tearing and interconnecting and its parallel solution algorithm. International Journal for Numerical Methods in Engineering, 32:1205-1227, 1991.

[17] C. Farhat and F.-X. Roux. Implicit parallel processing in structural mechanics. In J. T. Oden, editor, Computational Mechanics Advances, volume 2. North-Holland, June 1994.

[18] N. D. Hung and G. De Saxce. Frictionless contact of elastic bodies by finite element method and mathematical programming technique. Computers $\mathscr{E}$ Structures, 11:55-67, 1980.

[19] N. Kikuchi. Penalty/finite element approximations of a class of unilateral contact problems. In Penalty method and finite element method. ASME, New York, 1982.

[20] N. Kikuchi and J. T. Oden, editors. Contact problems in elasticity: a study of variational inequalities and finite element methods. SIAM, Philadelphia, 1988.

[21] A. Klarbring. A mathematical programming approach to three-dimensional contact problems with friction. Computer Methods in Applied Mechanics and Engineering, 58:175-200, 1986.

[22] A. Klarbring. Mathematical programming and augmented lagrangian methods for frictional contact problems. In Curnier [9], pages 409-422.

[23] J. Mandel and R. Tezaur. Convergence of a substructuring method with Lagrange multipliers. Numerische Mathematik, 73:473-487, 1996.

[24] J. Mandel, R. Tezaur, and C. Farhat. A scalable substructuring method by Lagrange multipliers for plate bending problems. SIAM Journal on Scientific and Statistical Computing, 36:1370-1391, 1999.

[25] H.-O. May. The conjugate gradient method for unilateral problems. Computers \& Structures, 12(4):595-598, 1986.

[26] B. Radi, O. A. Baba, and J. C. Gelin. Treatment of the frictional contact via a Lagrangian formulation. In Rodin and Shillor [30], pages 407-412.

[27] M. Raous and S. Barbarin. Conjugate gradient for frictional contact. In Curnier [9]. 
[28] M. Raous, M. Jean, and J.-J. Moreau, editors. Proceedings of the Second Contact Mechanics International Symposium, New York, 1995. Plenum Press.

[29] D. Rixen and C. Farhat. A simple and efficient extension of a class of substructure based preconditioners to heterogeneous structural mechanics problems. International Journal for Numerical Methods in Engineering, 46:501-534, 1998.

[30] E. Y. Rodin and M. Shillor, editors. Mathematical and computer modelling. Number 28. Pergamon Press, 1998.

[31] J. Simo and T. A. Laursen. An augmented Lagrangian treatment of contact problem involving friction. Computers \& Structures, 42:97-116, 1992.

[32] P. Wriggers. Finite element algorithms for contact problems. Archives of Computational Methods in Engineering, 2:1-49, 1995.

[33] P. Wriggers et al. Penalty and augmented Lagrangian formulations for contact problems. In Proc. NUMETA Conf., Swansea, 1985.

[34] Z. Zhong and J. Mackerle. Static contact problems - a review. Engineering Computations, 9:3-37, 1992. 